OPEN ACCESS

Edited by:

Claude Desplan,

New York University, United States

Reviewed by:

André Fiala,

University of Göttingen, Germany Mark A. Frye,

University of California, Los Angeles, United States

*Correspondence: Giovanni Bosco

giovanni.bosco@dartmouth.edu

Received: 18 February 2020 Accepted: 14 April 2020

Published: 08 May 2020

Citation:

Edwards KA, Hoppa MB and Bosco G (2020) The Photoconvertible Fluorescent Probe, CaMPARI, Labels Active Neurons in Freely-Moving Intact Adult Fruit Flies.

Front. Neural Circuits 14:22. doi: 10.3389/fncir.2020.00022

\section{The Photoconvertible Fluorescent Probe, CaMPARI, Labels Active Neurons in Freely-Moving Intact Adult Fruit Flies}

\author{
Katie A. Edwards ${ }^{1}$, Michael B. Hoppa ${ }^{2}$ and Giovanni Bosco ${ }^{1 *}$ \\ ${ }^{1}$ Department of Molecular and Systems Biology, Geisel School of Medicine, Dartmouth College, Hanover, NH, United States, \\ ${ }^{2}$ Department of Biological Sciences, Dartmouth College, Hanover, NH, United States
}

Linking neural circuitry to behavior by mapping active neurons in vivo is a challenge. Both genetically encoded calcium indicators (GECls) and intermediate early genes (IEGs) have been used to pinpoint active neurons during a stimulus or behavior but have drawbacks such as limiting the movement of the organism, requiring a priori knowledge of the active region or having poor temporal resolution. Calcium-modulated photoactivatable ratiometric integrator (CaMPARI) was engineered to overcome these spatial-temporal challenges. CaMPARI is a photoconvertible protein that only converts from green to red fluorescence in the presence of high calcium concentration and $405 \mathrm{~nm}$ light. This allows the experimenter to precisely mark active neurons within defined temporal windows. The photoconversion can then be quantified by taking the ratio of the red fluorescence to the green. CaMPARI promises the ability to trace active neurons during a specific stimulus; however, CaMPARI's uses in adult Drosophila have been limited to photoconversion during fly immobilization. Here, we demonstrate a method that allows photoconversion of multiple freely-moving intact adult flies during a stimulus. Flies were placed in a dish with filter paper wet with acetic acid $(\mathrm{pH}=2)$ or neutralized acetic acid $(\mathrm{pH}=7)$ and exposed to photoconvertible light (60 mW) for $30 \mathrm{~min}$ (500 ms on, $200 \mathrm{~ms}$ off). Immediately following photoconversion, whole flies were fixed and imaged by confocal microscopy. The red:green ratio was quantified for the DC4 glomerulus, a bundle of neurons expressing Ir64a, an ionotropic receptor that senses acids in the Drosophila antennal lobe. Flies exposed to acetic acid showed 1.3-fold greater photoconversion than flies exposed to neutralized acetic acid. This finding was recapitulated using a more physiological stimulus of apple cider vinegar. These results indicate that CaMPARI can be used to label neurons in intact, freely-moving adult flies and will be useful for identifying the circuitry underlying complex behaviors.

Keywords: optogenetics, CaMPARI, Drosophila melanogaster, photoconversion, behavior

\section{INTRODUCTION}

Calcium is an essential secondary messenger in neurons and has proven to be one of the most widely used signals to trace neural activity (Palmer and Tsien, 2006). Most neurons have a low intracellular calcium concentration of 50-100 $\mathrm{nM}$ that rises 10-100-fold higher during neural activation (Berridge et al., 2000), thus, an activity can be reliably marked by a spike in calcium concentration. 
Initially, measuring intracellular calcium was dependent on the delivery of dyes or fluorescent calcium indicators. While many of these indicators have good signal-to-noise ratios and fast kinetics (Paredes et al., 2008), they are typically only useful for a short period after delivery (Jia et al., 2011). Protein-based genetically encoded calcium indicators (GECIs) were developed to allow for multiple readings over multiple days (Looger and Griesbeck, 2012). Over the past two decades, GECIs have been continuously developed and modified to improve brightness (Tian et al., 2009; Chen et al., 2013; Barnett et al., 2017), kinetics (Chen et al., 2013; Sun et al., 2013), stability (Tian et al., 2009; Chen et al., 2013) and sensitivity (Chen et al., 2013; Barnett et al., 2017) thus enabling more efficient tracking of changes in activity over time in neuronal subpopulations and whole brains in a variety of model organisms.

Despite the broad success of GECIs in in vivo imaging, tracking neural activity with behavior is limited due to the necessity to head-fix organisms to obtain high-resolution images (Tian et al., 2009; Sun et al., 2013) except for the development of the miniscope for rodents and other small mammals (Aharoni and Hoogland, 2019). However, the field of view is often limited by the necessity to deliver light into the brain and may require a priori knowledge of the specific brain region relevant to the specific stimulus, regardless of whether the animal is head-fixed under a microscope or freely-moving with a miniscope (Aharoni and Hoogland, 2019; de Groot et al., 2020). Because some circuit activity is so short-lived, it may escape detection unless the experimenter has specific knowledge of where and when to image. As such, GECIs have limited use as a circuit-discovery tool in identifying activated circuits in freely behaving animals. This is especially true in non-transparent organisms or systems with non-transparent developmental stages. A potential solution to these challenges would be the ability to accurately "tag" or identify neurons during a defined stimulation period allowing detailed imaging after the activity. To do just that, Fosque et al. (2015) invented a calcium integrator, which combined a photoswitchable protein (mEOS) with molecular aspects of GCaMP, a GECI, to make photoswitching dependent on both calcium and violet light. With this new tool, active neurons expressing this integrator would convert fluorescent emission from green to red only in the simultaneous presence of high calcium concentration from neural activity and user-supplied violet light. This calcium integrator was named calcium-modulated photoactivatable ratiometric integrator (CaMPARI). Because of the transient nature of calcium and the user-defined photo-converting light, labeling with CaMPARI can be tightly controlled to track neural activity during a defined behavior.

Thus far CaMPARI has been successfully deployed in behaving solitary juvenile zebrafish (Moeyaert et al., 2018; Sha et al., 2019) and fruit fly larva (Patel and Cox, 2017) due to the translucent nature of those organisms. However, use in adult fruit flies has been limited due to the head cuticle of the fly. Most published reports of CaMPARI's uses in the adult fruit fly require the fly to be fixed or tethered on a microscope with direct illumination from a laser (Bohra et al., 2018; Manjila et al., 2019). While such experiments are valuable, they limit the movement of the fly and again require the animal to be solitary, which can affect how an animal responds to stimuli naturally (Ramdya et al., 2017). Thus, this is a central limitation of CaMPARI's versatility to identify circuits involved in natural behaviors and in large scale experiments on numbers of flies used in each experiment, precluding a tremendous benefit of the Drosophila system.

Here, we propose a setup that utilizes inexpensive and commonly used materials to take advantage of existing CaMPARI fly stocks to label neuronal activation in freely moving individuals or groups of flies. This involves the use of standard Petri dishes with passive humidification and a series of $405 \mathrm{~nm}$ LEDs that allow for photoconversion of CaMPARI in the freely-moving intact adult fly. With this setup, we demonstrate that photoconversion of specific neuronal circuits is possible in multiple sets of fruit flies simultaneously. We provide quantified photoconversion of an olfactory glomerulus known to be activated by exposure to acids (Ai et al., 2010) as a proof of principle that this method allows for quantifiable significant photoconversion through the intact head cuticle of the freely-moving adult fly. Because this method is scalable to any group size, we suggest that CaMPARI in Drosophila is easily adaptable for large scale genetic screens and/or discovery and mapping of novel circuits that are activated by the investigator's stimulus of choice.

This method has the benefits of photo-converting CaMPARI expressed in the brains of multiple flies at once, allowing for the full movement and exploration behaviors of the fly, including social and group behaviors. We suggest that this approach will be useful in studying ecologically relevant behaviors, neurogenetic factors, and the circuits that govern such behaviors.

\section{MATERIALS AND EQUIPMENTS}

\section{Fly Stocks}

UAS-CaMPARI-GAL4. $\mathrm{w}[*] ; \mathrm{P}\{\mathrm{y}[+\mathrm{mDint} 2] \mathrm{w}[+\mathrm{mC}]=\mathrm{UAS}-$ CaMPARI $\}$ attP40; PBac $\{\mathrm{y}[+\mathrm{mDint} 2] \mathrm{w}[+\mathrm{mC}]=$ GMR57C10GAL4\}VK00040

PBac $\{$ GMR57C10-GAL4\}VK00020

(Bloomington Drosophila Stock Center, catalog number: 58763).

UAS-CaMPARI. $\quad \mathrm{w}[*] ; \quad \mathrm{P}\{\mathrm{y}[+\mathrm{t} 7.7] \quad \mathrm{w}[+\mathrm{mC}]=\mathrm{UAS}-$ CaMPARI\}attP40 (Bloomington Drosophila Stock Center, catalog number 58761).

Ir64a-GAL4. $\mathrm{w}[*] ; \quad \mathrm{P}\{\mathrm{w}[+\mathrm{mC}]=\operatorname{Ir64a-GAL4.A}\} 183.8 ;$ TM2/TM6B, $\mathrm{Tb}[1]$ (Bloomington Drosophila Stock Center, catalog number 41732).

Ir64a RNAi. w1118; P\{GD2314\}v9011 (Vienna Drosophila Resource Center, catalog number 9011).

\section{Fly Crosses}

UAS-CaMPARI $x$ Ir64a-GAL4. 10 virgin female $\mathrm{w}[*] ; \mathrm{P}\{\mathrm{y}[+\mathrm{t} 7.7]$ $\mathrm{w}[+\mathrm{mC}]=$ UAS-CaMPARI $\}$ attP40 crossed with 5 male $\mathrm{w}[*]$; $\mathrm{P}\{\mathrm{w}[+\mathrm{mC}]=$ Ir64a-GAL4.A $\}$ 183.8; TM2/TM6B, Tb[1].

UAS-CaMPARI-GAL4 $\times$ Ir64a-RNAi (Ir64a knockdown in $\mathrm{nSyb}$ neurons). Ten virgin female $\mathrm{w}\left[{ }^{*}\right] ; \mathrm{P}\{\mathrm{y}[+\mathrm{mDint} 2]$ $\mathrm{w}[+\mathrm{mC}]=$ UAS-CaMPARI $\}$ attP40; PBac $\{\mathrm{y}[+\mathrm{mDint} 2]$ $\mathrm{w}[+\mathrm{mC}]=$ GMR57C10-GAL4 $\}$ VK00040 PBac\{GMR57C10GAL4\}VK00020 crossed with five male w1118; P\{GD2314\}v9011. 


\section{Solutions}

5\% (v/v) Acetic acid. Five millilter Glacial Acetic Acid (Fisher Chemical Acetic Acid, Glacial Lot\# 185624) diluted to $100 \mathrm{ml}$ with $\mathrm{H}_{2} \mathrm{O}$. For neutralized acetic acid, neutralized to $\mathrm{pH}=7$ with Sodium Hydroxide pellets (Thermo Fisher Scientific Lot\# 115786).

Apple Cider Vinegar. Bought from a local supermarket. For neutralized apple cider vinegar, neutralized to $\mathrm{pH}=7$ with Sodium Hydroxide pellets (Thermo Fisher Scientific Lot\# 115786).

Phosphate-buffered saline (PBS). Eighty gram Sodium Chloride (NaCl; EMD Chemicals SX0420-1 Lot\# UG25BZEMS), 2 g Potassium Chloride (KCl; Thermo Fisher Scientific P217-500 Lot\# 117211), 14.4 g Sodium Phosphate, Dibasic Anhydrous $\left(\mathrm{Na}_{2} \mathrm{HPO}_{4}\right.$; Fisher Chemical S374-500 Lot\# 151771), $2.4 \mathrm{~g}$ Potassium Phosphate, Monobasic $\left(\mathrm{KH}_{2} \mathrm{PO}_{4}\right.$; EMD Chemicals PX1565-1 Lot\# 2011061522) diluted to $1 \mathrm{~L}$ with $\mathrm{H}_{2} \mathrm{O}$, then sterilized by autoclave to make a $10 \times$ solution. $1 \times$ PBS is made by diluting $100 \mathrm{ml} 10 \times$ PBS to $1 \mathrm{~L}$ with $\mathrm{H}_{2} \mathrm{O}$.

PBT (PBS + 0.1\% Triton-X 100). One-hundred milliliter $10 \times$ PBS and $10 \mathrm{ml}$ Triton-X 100 (Sigma-Aldrich Lot\# MKBW8386V) diluted to $1 \mathrm{~L}$ with $\mathrm{H}_{2} \mathrm{O}$.

4\% formaldehyde in PBT. Two milliliter $16 \%$ formalehyde solution (w/v), Methanol-free (Thermo Fisher Scientific Lot\# UC2742481) and $6 \mathrm{ml} \mathrm{PBT.}$

Vectashield Antifade Mounting Medium (Vector Vectashield Lot\# ZC0829).

\section{Photoconversion Equipment}

Sanworks Pulse Pal v2 (Sanders and Kepecs, 2014; can be substituted with any pulse generator).

405 nm LEDs (Thorlabs M405L3).

Collimation Collars (Thorlabs SM1P25-A).

Current Controller (Thorlabs T-cube LED Driver LEDD1B).

Adaptor Plate for current controllers (KAP101).

Power Supply for current controllers (Thorlabs KCH601). Analog Handheld Power Meter Console (Thorlabs PM100A). Standard Photodiode Sensor for 400-1,100 nm (SC121C).

\section{Software}

Zeiss Zen Black 2.3.

Fiji (Schindelin et al., 2012).

Graphpad Prism 8.3.0.

\section{Other}

Petri dishes $(35 \times 10 \mathrm{~mm}$; Falcon 351007$)$.

42 Ashless Filter Paper (70 mm diameter; GE Healthcare Whatman 1442-070 Lot\# 16946862).

"PTFE" Printed 10-well 6 mm well diameter Slides (Electron Microscopy Sciences 63434-06 Lot\# 171020).

$20 \times 50$ mm No. 1 1/2 Coverslips (VWR 48393 194).

\section{Procedures}

\section{(A) Drosophila Cultures, Animal Manipulation, and Genetic Crosses}

Note: Drosophila melanogaster was used for all experiments described and culture conditions were used as described below.
One notable exception to standard fly manipulation was the use of ice to anesthetize adults, instead of the usual humidified carbon dioxide $\left(\mathrm{CO}_{2}\right)$. This was especially important for adult flies that were subsequently used in photoconversion experiments. Exposure to $\mathrm{CO}_{2}$ is neurotoxic and could activate olfactory as well as acid-sensing neurons while also possibly triggering a stress response (Suh et al., 2004; Ai et al., 2010). However, $\mathrm{CO}_{2}$ is still used for standard fly maintenance.

(1) Drosophila cultures were reared on standard cornmealmolasses media in plastic bottles (Genesse, catalog number $32-130$ ) at $22^{\circ} \mathrm{C}, \sim 40 \%$ humidity and $12 \mathrm{~h}$-dark/12 h-light cycle.

(2) Genetic crosses were necessary to achieve some genotypes of interest, for example where CaMPARI was being expressed in specific regions of the brain or when short-hairpin RNA (shRNA) was being expressed in addition to CaMPARI. Typically, 10 virgin females 4-6 days post-eclosion (dpe) were crossed with five males and placed in a $22 \mathrm{~mm}$ food vial (Genesee, catalog number 32-116). Adult parents were discarded after 4-7 days or transferred into a fresh food vial to continue laying eggs. Controlling the number of females and the number of days the females are allowed to lay eggs ensures that progeny are not overcrowded, thus ensuring maximum consistency in access to nutrition during development and adult body and tissue size. Adult progeny were collected for photoconversion 14-18 days after the initial cross was started.

- Virgin UAS-CaMPARI flies were crossed to Ir64a-GAL4 flies at 4 dpe and progeny collected at 1-2 dpe and isolated in groups of five females and three males at $25^{\circ} \mathrm{C}$.

- Pan-neuronal expressing CaMPARI flies were collected at 1-2 dpe and isolated in groups of five females and three males at $25^{\circ} \mathrm{C}$. Since this genotype was maintained as a stable stock it was not necessary to perform a genetic cross.

- To produce adult flies expressing CaMPARI and Ir64a-RNAi in nSyb-expressing neurons 10 virgin females were crossed to five UAS-Ir64a-RNAi males and progeny collected at 1-2 dpe and isolated in groups of five females and three males at $25^{\circ} \mathrm{C}$.

\section{(B) Photoconversion Setup (Duration: 1 day)}

The setup can be adjusted to suit the needs of the investigator/experiment. In Figure 1, we show our setup of 16 LEDs (Thorlabs M405L3) with collimation collars (Thorlabs SM1P25-A) adjusted to match the area of the Petri dishes. Each LED is connected to a current controller (Thorlabs T-Cube LED Driver LEDD1B) where the power of the LED can be adjusted via the current while the power can be measured for each LED using an optical power meter (Thorlabs PM100A) and a standard photodiode sensor (SC121C). The current controllers can be powered individually but are here powered by a power supply (KCH601) with an adaptor plate (KAP101). Each current controller is connected to a pulse generator (Sanworks Pulse Pal v2) with standard female BNC to female BNC cables.

The PulsePal can be programmed to control the on/off cycle of the LEDs. The PulsePal has four "output channels" that can be 


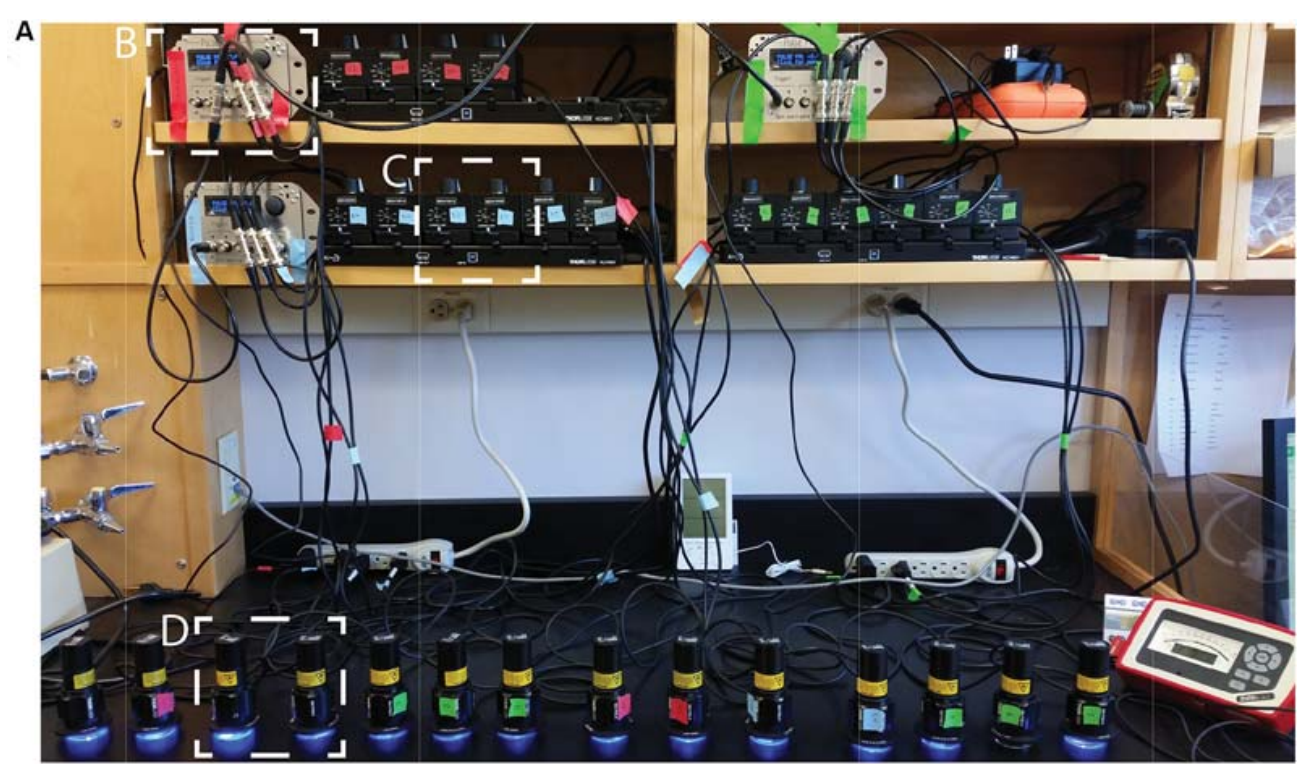

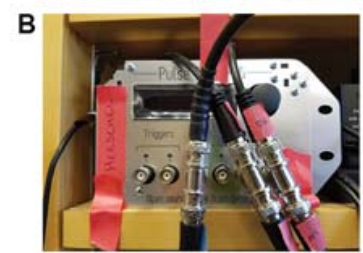

(B) Pulse Generator Controls light on/off cycle

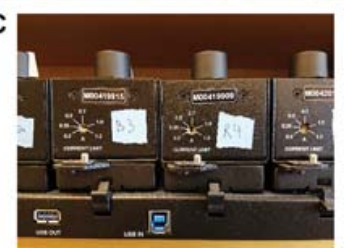

(C) Current Controller Controls LED Power

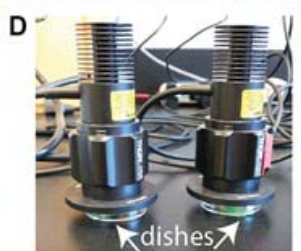

(D) LED

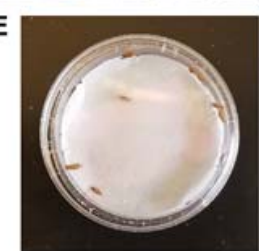

(E) Petri Dish with Flies

FIGURE 1 | Photoconversion setup. A view of the setup as used to induce photoconversion in freely moving flies. (A) Sanworks Pulse Pal v2 is used to control the LED on/off cycle. (B) The pulse generators are connected to current controllers (Thor Labs M00420201). The knobs of the current controllers are used to set the LED power. (C) The $405 \mathrm{~nm}$ LEDs (Thor Labs M405L3) light is dispersed with collimation collars (Thor Labs SM1P25-A) set to match the area of the dishes. (D,E) Petri dishes are prepared with moistened filter paper and can contain individual or sets of adult Drosophila that sit underneath the LEDs.

programmed separately and triggered simultaneously by linking them to the same trigger channel.

\section{(C) Photoconversion of Flies (Duration: $1 \mathrm{~h}$ )}

(1) Prepare the Petri dishes that will contain the flies during photoconversion. Trim ashless filter paper by trimming to the size of the Petri dish. Moisten the filter paper by pipetting directly onto the paper $150 \mu \mathrm{l}$ PBS or other solutions to be used as stimuli. Drops should be evenly distributed so that the entire filter paper is moistened. The paper should be pushed flat to the bottom of the dish so that flies cannot get into crevices underneath the paper. PBS can be substituted with solutions to be used as stimuli. Here, as experimental conditions, we used 5\% acetic acid and apple cider vinegar solutions in place of PBS.

Critical step: It is necessary to introduce a solution to the dish to control the humidity within the dish. Without added solution, the flies can overheat and desiccate, killing them during photoconversion.

(2) Place vials containing flies on ice for $20 \mathrm{~min}$. Vials are partially submerged in a bucket containing ice.
Note: Certain anesthetizing steps may induce neural activation, as described above.

(3) Calibrate LED power by adjusting the dial of the current controller using a power meter. In this study, we used $75 \mathrm{~mW} / \mathrm{cm}^{2} \pm 0.5 \mathrm{~mW} / \mathrm{cm}^{2}$ power.

Note: LED power may need to be adjusted for deeper brain regions or neurons with less frequent firing rates.

(4) Setup a pulse generator (Figure 1). The Pulse Pal was programmed to deliver monophasic pulses of $5 \mathrm{~V}$ every $500 \mathrm{~ms}$ with a duty cycle of $200 \mathrm{~ms}$ until manually aborted.

Note: In our setup, we used open-source hardware, the Sanworks Pulse Pal, however, any pulse generator can be used that is compatible with the LEDs.

(5) From the anesthetized fly vials that were placed on ice, dispense five female and three male adults (4-6 dpe) into each dish prepared in Step 1 and place lids on each dish.

Critical step: Wait until flies are actively moving on their own before moving to the next step. This ensures they have fully recovered from anesthesia. 
(6) Place LEDs on top of dishes and set PulsePal/pulse generator to "Continuous." Allow the program to continue for $30 \mathrm{~min}$ or the desired time.

(7) Prepare fixation reagents (4\% formaldehyde in PBT) and dispense in $800 \mu \mathrm{l}$ aliquots in 1.5 microcentrifuge tubes.

(8) End photoconversion protocol. Anesthetize the flies by delivering $\mathrm{CO}_{2}$ into the space between the dish and the lid using a syringe. Transfer the whole flies directly into the $1.5 \mathrm{ml}$ tubes containing a fixation solution. Spin down the tubes containing flies in a centrifuge for $5 \mathrm{~min}$ at 13,000 rpm. Store flies in fixation solution at $4^{\circ} \mathrm{C}$ for $14-18 \mathrm{~h}$.

Note: Flies should be protected from light.

Note: Alternatively, fixation is not necessary as the tissue could be imaged live or dissected and then fixed. CaMPARI is sensitive to fixation and so signal, particularly red signal, is compromised (Fosque et al., 2015). Keep in mind the turnover rate of photoconverted CaMPARI, which is $\sim 5 \mathrm{~h}$, when performing live imaging or dissections. Recently an antibody has been developed that specifically binds the red CaMPARI that can be used to amplify the signal (Moeyaert et al., 2018).

\section{(D) Brain Dissection and Tissue Sample Preparation} (Duration: 2 h)

(1) Dissect the brain from the head cuticle, as previously described (Wu and Luo, 2006) into the wells of a glass spot plate.

(2) Wash dissected brains three times for $10 \mathrm{~min}$ by pipetting $150 \mu \mathrm{l}$ PBT into each well containing the tissue.

(3) Wash dissected brains four times for $5 \mathrm{~min}$ by pipetting $150 \mu \mathrm{l}$ PBS into each well containing the tissue.

(4) Dispense Vectashield Antifade Mounting Medium into each well of a slide enough to just fill the well.

(5) Carefully transfer dissected brains into the wells of the slide. Each well should contain no more than three brains.

Critical step: Brains should be oriented such that the ROI is closest to the coverslip to prevent light dispersal through tissue and obtain better resolution images.

(6) Mount brain tissue on slides with Vectashield Antifade Mounting Medium.

Note: This mounting medium remains a liquid on the slide and it's recommended to seal the slide.

\section{(E) Imaging (Duration: 2-8 h)}

Images were collected using a Zeiss LSM 880 microscope and Zeiss Zen Black 2.3 software using a $20 \times$ Plan-apochromatic (0.8 NA) objective. $488 \mathrm{~nm}$ and $561 \mathrm{~nm}$ laser lines were used for illumination and a GaAsP detector for imaging. $1,024 \times 1,024$ images were collected with a $1.02 \mu$ s pixel dwell and 16-bit depth. Z-stacks were collected at a $0.97 \mu \mathrm{m}$ step.

\section{(F) Image Analysis (Duration: 2-6 h)}

(1) For each sample, identify slices that contain the ROIs using Fiji.

(2) Combine slices and collapse into a single image using an average intensity projection (AIP; Figure $2 \mathrm{C}$ ).

(3) Use the "polygon tool" to trace the ROI within the AIP in the green channel, as in "DC4" in Figure 2C.
(4) Copy the ROI and move to a region outside the imaged brain, as in "bgrd" in Figure 2C. This ROI will be used for background subtraction in the green channel.

(5) Copy the same ROIs to the AIP of the red channel.

(6) Use the "Measure" function to calculate the average intensity of each ROI.

(7) Subtract background fluorescence from each ROI. For example, the average intensity of "bgrd" ROI is subtracted from the average intensity of the "DC4" ROI.

(8) Divide background-subtracted red channel fluorescence by background-subtracted green channel fluorescence to determine the ratio.

(9) Repeat steps 3-8 for any additional ROIs in the brain.

(10) Red:green ratios were normalized to the ratios of images from flies exposed to $\mathrm{pH}=7$ solutions by grouping flies that were photoconverted simultaneously.

Critical step: It is important to only compare images that were taken on the same day to avoid batch effects. Microscope laser power can vary day-to-day and affect the quantification of the images.

\section{(G) Statistics}

All data are presented as means \pm SEM. Comparisons between two groups were performed using a Mann-Whitney $\mathrm{U}$ test in Graphpad Prism 8.3.0.

\section{RESULTS}

\section{Acetic Acid Exposure Results in Specific Photoconversion of the DC4 Glomerulus in Flies Expressing CaMPARI in Ir64a-Expressing Neurons}

We first demonstrated that we could get specific photoconversion in acid-sensing neurons through the head cuticle of freely-moving adult flies by exposing flies with pan-neuronally expressing CaMPARI to 5\% acetic acid (AA; $\mathrm{pH}=2)$. Previously it was shown that Ir64a-expressing neurons of the DC4 glomerulus (Figure 2A) are specifically activated by acids (Ai et al., 2010). Adult intact freelymoving flies were exposed to $5 \% \mathrm{AA} \mathrm{pH}=2$ for $30 \mathrm{~min}$ while also being exposed to photoconvertible light $(405 \mathrm{~nm}$; $500 \mathrm{~ms}$ on, $200 \mathrm{~ms}$ off). This resulted in a photoconverted region within each antennal lobe consistent with the DC4 glomerulus (Figure 2B).

To confirm this photoconverted region was specific to the acid-sensing neurons of the DC4 glomerulus, we limited expression of CaMPARI to the DC4 glomerulus by driving expression of CaMPARI with an Ir64a GAL4 driver (Figures 2C-F). Intact, freely-moving adult flies were exposed to $5 \% \mathrm{AA}(\mathrm{pH}=2)$ or neutralized $5 \% \mathrm{AA}(\mathrm{pH}=7)$ for $30 \mathrm{~min}$ while simultaneously exposed to photoconvertible light. Average intensity projection (AIP) images of the full fly brain are shown for $5 \% \mathrm{AA}(\mathrm{pH}=2$; Figure $2 \mathrm{C})$ and $5 \% \mathrm{AA}(\mathrm{pH}=7$; Figure 2D). As shown in the green channel, the expression of CaMPARI is limited to the DC4 glomerulus. The signal of photoconverted CaMPARI is present for $5 \% \mathrm{AA}(\mathrm{pH}=2)$, but not when the 


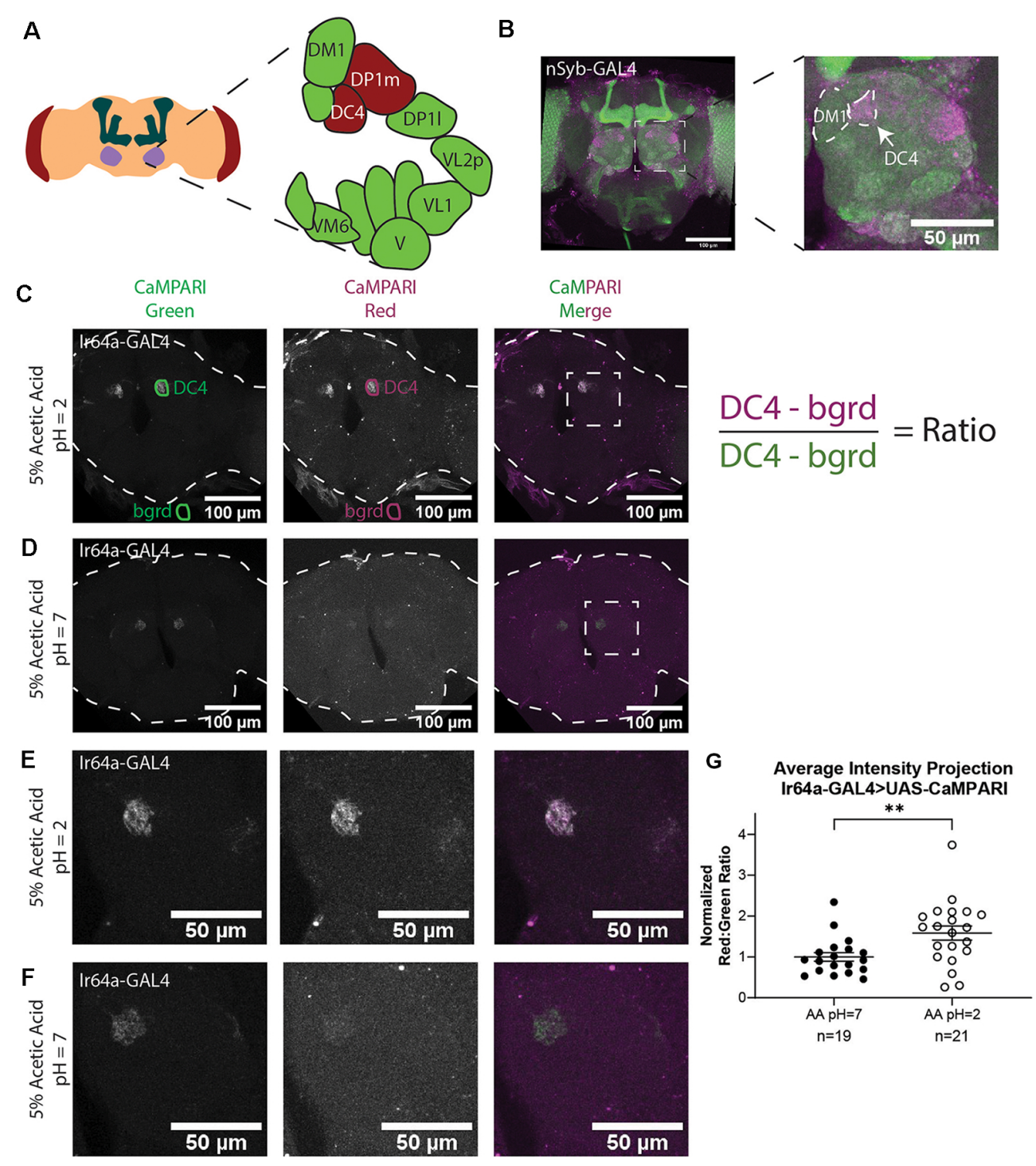

FIGURE 2 | Analysis of images of flies expressing CaMPARI. (A) Schematic of the Drosophila head labeling the antennal lobe and antennal lobe glomeruli in the region of interest (ROI). Acid-sensing glomeruli (DC4 and DP1 m) are labeled in red. (B) Representative maximum intensity projection (MIP) of adult fly brain expressing CaMPARI pan-neuronally exposed to $5 \%$ acetic acid at $\mathrm{pH}=2$ for $30 \mathrm{~min}$ and imaged at 20x magnification. A white dotted square indicates a single antennal lobe blown up to the right demonstrating photoconversion of DC4 glomerulus. The DM1 glomerulus is used as a landmark to find the DC4 glomerulus. (C,D) Representative average intensity projection (AIP) images of adult fly brains expressing CaMPARI in Ir64a-expressing neurons exposed to 5\% acetic acid (AA) at $\mathrm{pH}=2$ (C) or pH = 7 (D) for 30 min and imaged at 20x magnification. An ROI was drawn around the DC4 glomeruli in the green channel (DC4) and then copied and moved to the background (bgrd) to background-subtract the average green intensity in the DC4 glomerulus. The same pair of ROls were copied to the red channel to collect the background-subtracted average red intensity. The ratio was then collected by dividing the red intensity by the green intensity as shown on the right. Photoconversion is seen in the red channel in panel (B) when $\mathrm{pH}=2$ but not in panel (C) when $\mathrm{pH}=7$. The antennal lobes are blown up in panels (E,F) as designated by the white dotted rectangle. (E,F) Representative maximum intensity projection (MIP) images of the adult fly antennal lobes expressing CaMPARI in Ir64a-expressing neurons exposed to $5 \% \mathrm{AA}$ at $\mathrm{pH}=2$ (E) or $\mathrm{pH}=7$ (F) for 30 min. (G) The Red:Green ratio of flies exposed to $\mathrm{AA}$ of $\mathrm{pH}=2$ and $\mathrm{pH}=7$ for 30 min. Flies exposed to $\mathrm{AA} \mathrm{pH}=2(n=21)$ have 1.6-fold greater photoconversion compared to flies exposed to $\mathrm{AA} \mathrm{pH}=7(n=19)$. ${ }^{* *} P=0.0064$, Mann-Whitney $U$-test.

acetic acid has been neutralized. Maximum intensity projection (MIP) of a single antennal lobe, as designated by the white dotted box in Figures 2C,D, are shown for both 5\% AA ( $\mathrm{pH}=2$; Figure 2E) and neutralized AA ( $\mathrm{pH}=7$; Figure 2F). These results confirm that photoconversion of CaMPARI in intact, freely-moving adult flies exposed to acetic acid is specific to the DC4 glomerulus.

\section{Photoconversion of CaMPARI Can Be Quantified in Freely-Moving Intact Adult} Flies

One of the major benefits of using CaMPARI as a tool for tracing active neurons is its use as a ratiometric integrator, meaning the level of photoconversion can be quantified by 
taking the ratio of CaMPARI intensity in the red channel to the CaMPARI intensity in the green channel. We quantified the red:green ratios of average intensity projection (AIP) images of freely-moving intact adult flies expressing CaMPARI in Ir64aexpressing neurons exposed to $5 \%$ acetic acid (AA; $\mathrm{pH}=2$; Figure $2 \mathrm{C})$ or neutralized $5 \% \mathrm{AA}(\mathrm{pH}=7$; Figure 2D). Five sets of 3-5 flies were exposed to acetic acid or neutralized acetic acid for $30 \mathrm{~min}$ while simultaneously exposed to photoconvertible light (405 nm; $500 \mathrm{~ms}$ on, $200 \mathrm{~ms}$ off). Immediately following photoconversion, flies were fixed in $4 \%$ formaldehyde in PBS + $0.1 \%$ TritonX-100 overnight. Brains were dissected from female flies and mounted on slides and imaged by laser-scanning confocal microscopy.

AIP images were constructed with Fiji using Z-project for all slices containing CaMPARI expression. The "polygon tool" was used to draw a region of interest (ROI) around each glomerulus in the green channel as shown in Figures 2C,D ("DC4"). These ROIs were saved to the ROI Manager in Fiji then each ROI was copied and moved to a region outside the fly brain to collect a background measurement ("bgrd") for the green channel. All four of these ROIs were then copied to the red channel. The ratio was then calculated as the "DC4" intensity of the red channel minus red channel "bgrd" intensity divided by the "DC4" intensity of the green channel minus the green channel "bgrd" intensity. This resulted in two ratios for each fly brain, one for the right "DC4" glomeruli and a second for the left. Because these ratios were always comparable they were averaged to give a single datum for each fly brain (Supplementary Figure S1). Each ratio was then normalized to the average ratio of flies exposed to $5 \% \mathrm{AA}(\mathrm{pH}=7)$ for each set that was photoconverted and imaged on the same day to minimize batch effects. We found that flies with exposure to $5 \% \mathrm{AA}(\mathrm{pH}=2 ; n=19$ individual flies) had 1.6-fold photoconversion compared to flies exposed to $5 \% \mathrm{AA}(\mathrm{pH}=7 ; n=21$ individual flies) in the DC4 glomerulus (Figure 2G).

To confirm measurements taken from AIP images accurately reflect each slice of the ROI, ROIs were drawn for every slice and averaged together. Despite a change in the area of the ROI in individual slices, these ratios were not appreciably different from the ones measured from AIP images (Supplementary Figure S2), thus we continued to use only AIP images to quantify photoconversion. These results confirm we can use AIP images to quantify the photoconversion ratio of an ROI in the fly brain when CaMPARI is photoconverted in the freely-moving intact adult fly.

\section{Photoconversion of CaMPARI Is Comparably Quantified in Freely-Moving Intact Flies Expressing Pan-Neuronal CaMPARI}

While it is useful to limit the expression of CaMPARI to subsets of neurons to obtain photoconversion ratios, for behaviors that recruit multiple regions of interest it may be more useful to quantify CaMPARI when it is expressed pan-neuronally. We wanted to confirm that ratios calculated from AIP images of pan-neuronally expressed CaMPARI would be comparable to the ratios we calculated when CaMPARI expression was limited to Ir64a-expressing neurons. Synaptobrevin (nSyb) is a presynaptic protein and expressing CaMPARI with a nSyb GAL4 driver allows CaMPARI to be expressed in all neurons expressing nSyb without limiting expression to presynaptic terminals. Five sets of three to five flies expressing CaMPARI driven by a nSyb GAL4 driver were exposed to $5 \%$ acetic acid or neutralized $5 \%$ acetic acid for $30 \mathrm{~min}$ while simultaneously exposed to photo-converting light (405 nm; $500 \mathrm{~ms}$ on, $200 \mathrm{~ms}$ off) and their brains dissected and imaged as described above. Maximum intensity projection (MIP) images of one antennal lobe are shown in Figure 3 for flies exposed to either $5 \%$ AA $(\mathrm{pH}=2$; Figure 3A) or neutralized 5\% AA ( $\mathrm{pH}=7$; Figure 3B). Ratios were calculated from AIP images as described above and by identifying first the DM1 glomerulus on the medial edge of the antennal lobe and tracing the DC4 glomerulus adjacent to it (Figure 2B). We found freely-moving intact adult flies with pan-neuronally expressed CaMPARI exposed to $5 \% \mathrm{AA}(\mathrm{pH}=2$; $n=20$ individual flies) had 1.6-fold photoconversion compared to flies exposed to $5 \% \mathrm{AA}$ ( $\mathrm{pH}=7 ; n=20$ individual flies) in the DC4 glomerulus (Figure 3E), the same fold photoconversion as demonstrated in flies expressing CaMPARI in Ir64a-expressing neurons. This suggests that comparable photoconversion ratios can be calculated from either subset of neurons expressing CaMPARI or pan-neuronally expressing CaMPARI.

We wanted to evaluate how are fixation protocol affected the photoconversion ratio because fixation is known to quench the fluorescence of red CaMPARI. Immediately following $30 \mathrm{~min}$ of photoconversion, three sets of 4-5 flies were anesthetized on ice and the brain was dissected and mounted on slides with Vectashield, then imaged as described above. Ratios were calculated from AIP images as described above. Each ratio was then normalized to the average ratio of flies exposed to $5 \% \mathrm{AA}$ $(\mathrm{pH}=7)$ for each set that was photoconverted and imaged on the same day to minimize batch effects. We found that flies with exposure to $5 \% \mathrm{AA}$ ( $\mathrm{pH}=2 ; n=14$ individual flies) had 1.4 -fold photoconversion compared to flies exposed to $5 \%$ AA $(\mathrm{pH}=7$; $n=15$ individual flies) in the DC4 glomerulus (Supplementary Figure S3). While imaging live tissue did seem to increase the fluorescence of red CaMPARI, it also increased the fluorescence of green CaMPARI, resulting in a decrease of the ratio. The ratios between these two methods are comparable so either method could be used.

We also wanted to evaluate the amount of time the brain is photoconverted. In addition to $30 \mathrm{~min}$, we photoconverted for $5 \mathrm{~min}$ or $15 \mathrm{~min}$. Ratios were calculated from AIP images as described above. We found that flies with exposure to $5 \%$ AA $(\mathrm{pH}=2)$ for $5 \mathrm{~min}(n=23$ individual flies) had 0.8 -fold photoconversion compared to flies exposed to $5 \% \mathrm{AA}(\mathrm{pH}=7$; $n=22$ individual flies) in the DC4 glomerulus (Supplementary Figure S4). Flies with exposure to $5 \%$ AA $(\mathrm{pH}=2)$ for 15 min ( $n=23$ individual flies) had 1.3-fold photoconversion compared to flies exposed to $5 \% \mathrm{AA}$ ( $\mathrm{pH}=7 ; n=20$ individual flies). Photoconversion for $30 \mathrm{~min}$ had the highest ratio so we continued to use this time for all experiments.

To further confirm the specificity of photoconversion in flies expressing pan-neuronal CaMPARI, these flies were crossed with 

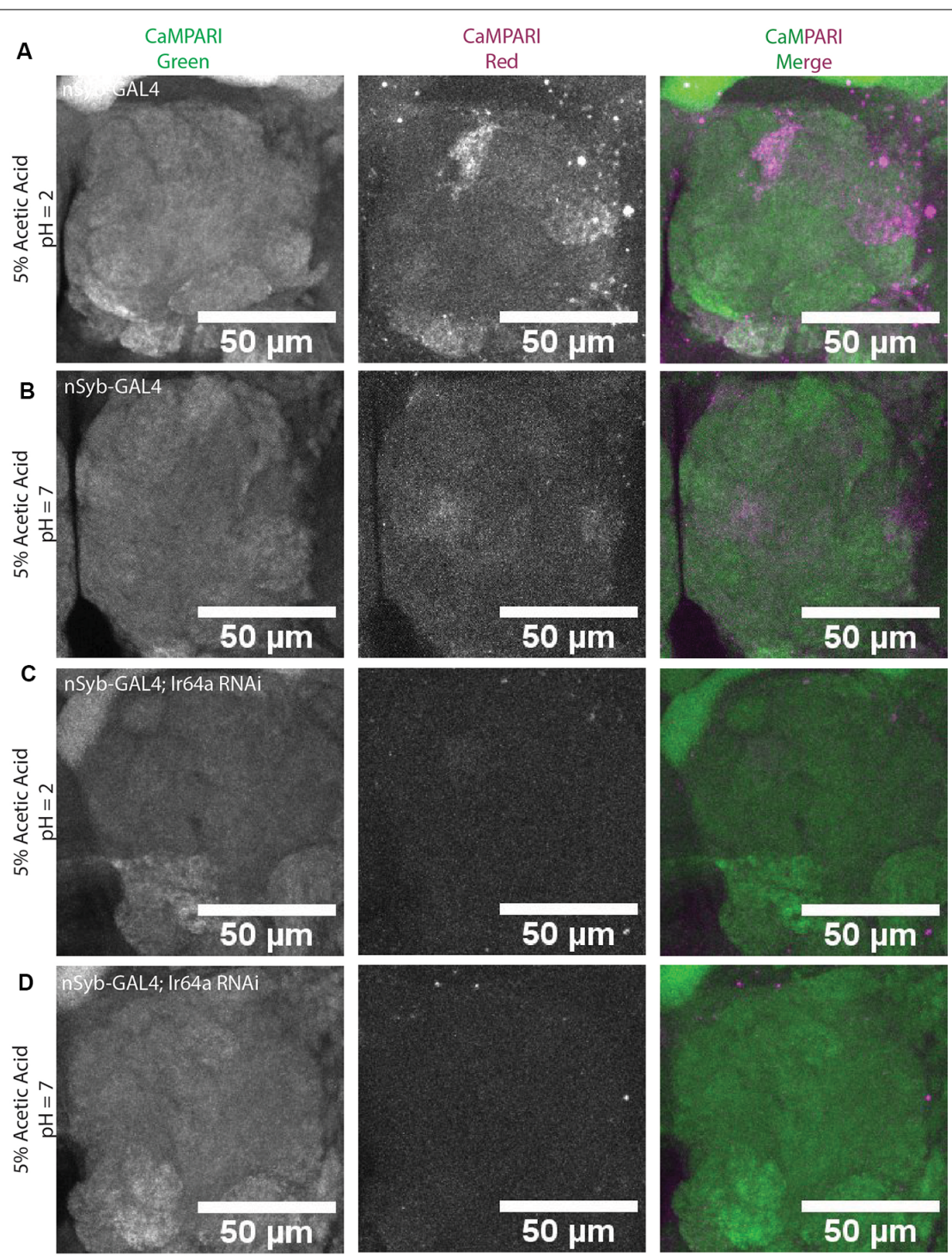

E Average Intensity Projection nSyb-GAL4>UAS-CaMPARI

F Average Intensity Projection nSyb-GAL4>UAS-CaMPARI nSyb-GAL4>Ir64a RNAi
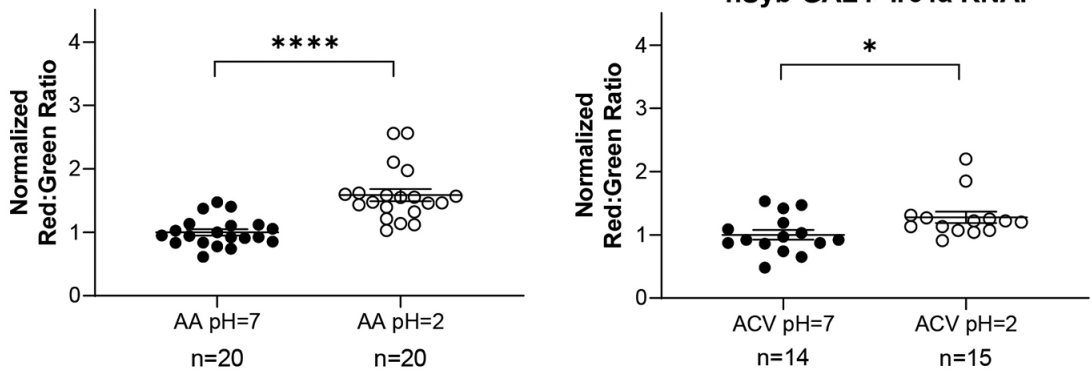

FIGURE 3 | Flies with Pan-neuronally expressed CaMPARI have comparable measurable Photoconversion in flies exposed to acetic acid. (A,B) Representative MIP images of adult fly antennal lobe expressing CaMPARI in nSyb-expressing neurons exposed to $5 \%$ acetic acid (AA) at pH $=2$ (A) or pH $=7$ (B) for 30 min. Photoconversion is seen in the red channel in panel (A) when $\mathrm{pH}=2$ but not in panel (B) when $\mathrm{pH}=7$. (C,D) Representative MIP images of the adult fly antennal lobe expressing CaMPARI and Ir64a RNAi in nSyb-expressing neurons exposed to $5 \% \mathrm{AA}$ at $\mathrm{pH}=2$ (C) or $\mathrm{pH}=7$ (D) for 30 min. Photoconversion is not seen in the red channel when the $\mathrm{pH}=2$ (C) or $\mathrm{pH}=7$ (D). (E) The Red:Green ratio of flies exposed to $\mathrm{AA}$ of $\mathrm{pH}=2$ and $\mathrm{pH}=7$ for 30 min. Flies exposed to $\mathrm{AA} p \mathrm{pH}=2(n=20)$ have 1.6-fold greater photoconversion compared to flies exposed to AA pH $=7(n=20)$. $* * * *<0.0001$, Mann-Whitney $U$-test. (F) The Red:Green ratio of flies exposed to $\mathrm{AA}$ of $\mathrm{pH}=2$ and $\mathrm{pH}=7$ for $30 \mathrm{~min}$. Flies exposed to $\mathrm{AA} \mathrm{pH}=2(n=14)$ have 1.3-fold greater photoconversion compared to flies exposed to $\mathrm{AA}$ pH $=7$ $(n=15) .{ }^{*} P=0.0151$, Mann-Whitney U-test. 
a line capable of expressing an shRNA directed at Ir64a mRNA, thus triggering RNA interference (RNAi), to induce knockdown of the acid-sensing Ir64a receptor. Three sets of four to five flies were exposed to $5 \%$ acetic acid or neutralized 5\% acetic acid for $30 \mathrm{~min}$ while simultaneously exposed to photoconvertible light ( $405 \mathrm{~nm} ; 500 \mathrm{~ms}$ on, $200 \mathrm{~ms}$ off). The brains were then dissected and imaged as described above. Ratios were calculated from AIP images as described above. We found that freely-moving intact adult flies with pan-neuronally expressing CaMPARI and Ir64a RNAi exposed to 5\% AA ( $\mathrm{pH}=2 ; n=14$ individual flies; Figure 3C) had 1.3-fold photoconversion compared to flies exposed to $5 \%$ AA ( $\mathrm{pH}=7 ; n=15$ individual flies; Figure 3D) in the DC4 glomerulus (Figure 3F). Even with knockdown of Ir64a, the amount of photoconversion in flies exposed to 5\% AA $(\mathrm{pH}=2)$ is still significantly increased compared to flies exposed to neutralized AA, however, the amount of photoconversion is reduced by $50 \%$ compared to flies not expressing the Ir64a RNAi. This is probably due to incomplete knockdown of the receptor. Still, this reduction in photoconversion suggests the photoconversion seen when flies are exposed to $5 \% \mathrm{AA}$ $(\mathrm{pH}=2)$ is specific to the activation of neurons expressing the Ir64a receptor.

\section{Apple Cider Vinegar Exposure Results in Specific Photoconversion of the DC4 Glomerulus in Flies Expressing CaMPARI}

Next, we wanted to compare the photoconversion we demonstrated with $5 \%$ acetic acid to a similar physiologicallyrelevant stimulus, apple cider vinegar, which is typically attractive to Drosophila melanogaster. Five to six sets of three to five flies expressing CaMPARI driven by a synaptobrevin (nSyb) GAL4 driver or an Ir64a GAL4 driver were exposed to apple cider vinegar (ACV) or neutralized ACV for $30 \mathrm{~min}$ while simultaneously exposed to photoconvertible light (405 nm; $500 \mathrm{~ms}$ on, $200 \mathrm{~ms}$ off) and their brains dissected and imaged as described above. Maximum intensity projection (MIP) images of one antennal lobe are shown in Figure 4 for flies exposed to either $5 \%$ AA $(\mathrm{pH}=2$; Figures 4 A,C) or neutralized $5 \%$ AA ( $\mathrm{pH}=7$; Figures 4B,D). Ratios were calculated from AIP images as described above. We found that freely-moving intact adult flies with pan-neuronally expressing CaMPARI exposed to ACV ( $\mathrm{pH}=2 ; n=27$ individual flies) had 2.1-fold photoconversion compared to flies exposed to neutralized ACV ( $\mathrm{pH}=7 ; n=21$ individual flies) in the DC4 glomerulus (Figure 4E). Similarly, flies expressing CaMPARI specifically in Ir64a-expressing neurons exposed to ACV ( $\mathrm{pH}=2$; $n=19$ individual flies) had 3.1-fold photoconversion compared to flies exposed to neutralized ACV ( $\mathrm{pH}=7 ; n=18$ individual flies; Figure 4F).

\section{DISCUSSION}

Previously, researchers have used intermediate early genes (IEGs) in mammals, such as Arc (Link et al., 1995; Guzowski et al., 1999) and cFos (Cole et al., 1989) as an indirect measure of neuronal activation, however, the use of IEGs is limited by the transient nature of their expression and lack of temporal resolution (Fields et al., 1997). In Drosophila, many IEGs are not conserved and the use of IEGs was limited to evaluating the expression of Hr38 (Fujita et al., 2013; Adhikari et al., 2019). Olfactory glomeruli were previously mapped in freelymoving flies using 2-deoxyglucose autoradiography (Rodrigues and Buchner, 1984). This method involved injecting flies and had non-quantifiable signal-to-noise, but laid the groundwork for follow-up with genetically encoded calcium indicators (GECIs), such as GCaMP (Riemensperger et al., 2012). GCaMP is a powerful tool for visualizing immediate activation but usually limits the movement of the organism during imaging and also limits the field of view during imaging. CaMPARI was developed as a solution to these limitations, however, methods requiring the adult fly to be fixed underneath a microscope have continued to limit its usefulness in studying the complex behaviors of adult Drosophila.

We have developed a method to trace active neural circuitry in freely-moving intact adult Drosophila. While previous methods have used direct laser light from microscopes, requiring immobilization of the fly (Bohra et al., 2018; Manjila et al., 2019), our method using LEDs and Petri dishes allows the flies to remain mobile and freely behaving and allows us to capture the active circuitry of multiple flies simultaneously. We demonstrate that this method faithfully recapitulates a previous study (Ai et al., 2010) demonstrating that exposure to acids activates the Ir64aexpressing neurons of the DC4 glomerulus in the antennal lobe of adult flies.

Using CaMPARI specifically expressed in Ir64a-expressing neurons while exposing flies to acetic acid, we showed that we can label active neurons in freely-moving adult flies and quantify the photoconversion in the DC4 glomerulus. The red:green ratio of flies exposed to acetic acid was significantly higher than flies exposed to neutralized acetic acid. Additionally, we showed that photoconversion between the left and right sides of the brain was fairly consistent, suggesting that light penetration from the LED was evenly distributed to both sides of the head. We were able to recapitulate these results with a pan-neuronally expressed CaMPARI. Quantification of the photoconversion in these flies was equivalent to the photoconversion seen when CaMPARI expression was limited to Ir64a-expressing neurons. This indicates CaMPARI can be used quantitatively whether CaMPARi expression is limited to subsets of neurons or is expressed broadly. CaMPARI expression is only limited by the strength and specificity of available driver lines. The ability to faithfully label active neurons with pan-neuronally expressing CaMPARI suggests CaMPARI can be used to label active circuitry even without a priori knowledge of which neurons should be active with a stimulus. Thus, our method allows for CaMPARI to be used as an exploratory tool in freely moving flies to discover novel parts of a circuit.

While our results show promise for CaMPARI as an exploratory tool in freely moving flies, there remain some caveats that may be addressed as the tool continues to develop. For example, the signal of red CaMPARI can be relatively 

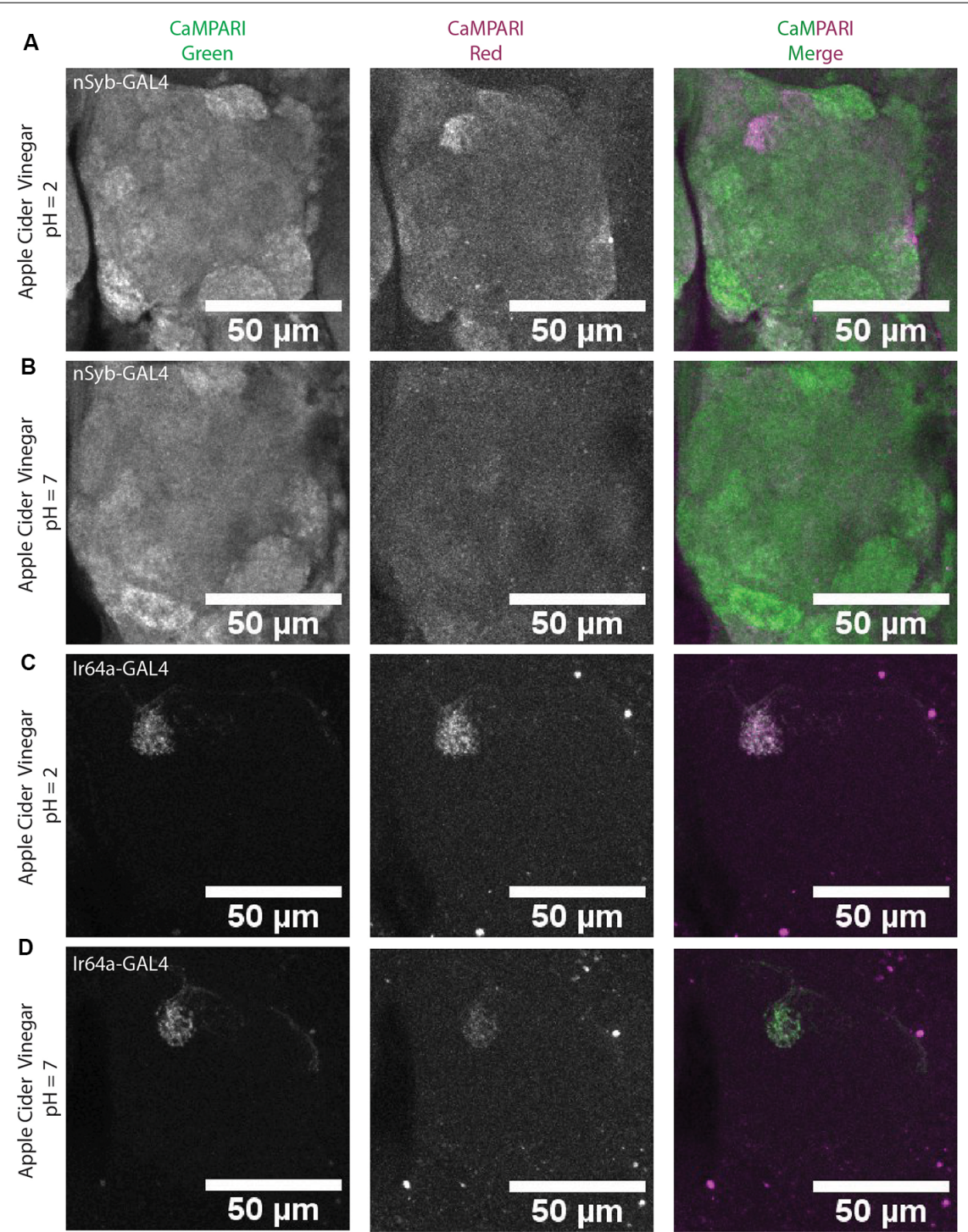

E Average Intensity Projection

F Average Intensity Projection nSyb-GAL4>UAS-CaMPARI
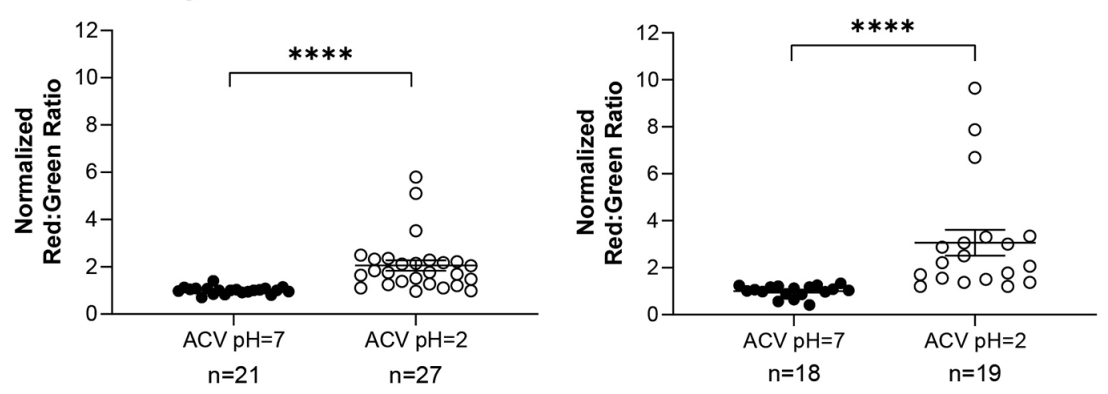

FIGURE 4 | Flies expressing CaMPARI have measurable photoconversion when exposed to apple cider vinegar. (A,B) Representative maximum intensity projection $(\mathrm{MIP})$ images of the adult fly antennal lobe expressing CaMPARI in $\mathrm{nSyb}$-expressing neurons exposed to $5 \%$ apple cider vinegar $(\mathrm{ACV})$ at $\mathrm{pH}=2 \mathbf{( A )}$ or $\mathrm{pH}=7 \mathbf{( B )}$ for $30 \mathrm{~min}$. Photoconversion is present in Ir64a-expressing neurons in panel $\mathbf{( A )}$ when the $\mathrm{pH}=2$, however, no appreciable photoconversion is seen in panel (B) when the $\mathrm{pH}=7$. (C,D) Representative MIP images of the adult fly antennal lobe expressing CaMPARI in Ir64a-expressing neurons exposed to $5 \%$ ACV at pH $=2$ (C) or $\mathrm{pH}=7$ (D) for 30 min. Photoconversion is seen in the red channel in panel (C) when $\mathrm{pH}=2$ but not in panel (D) when pH = 7. (E) The Red:Green ratio of flies exposed to $\mathrm{ACV}$ of $\mathrm{pH}=2$ and $\mathrm{pH}=7$ for $30 \mathrm{~min}$. Flies exposed to $\mathrm{ACV} \mathrm{pH}=2(n=27)$ have 2.1-fold greater photoconversion compared to flies exposed to ACV $\mathrm{pH}=7(n=21) .{ }^{* * * *} P<0.0001$, Mann-Whitney $U$-test. (F) The Red:Green ratio of flies exposed to $\mathrm{ACV}$ of $\mathrm{pH}=2 \mathrm{and} \mathrm{pH}=7$ for 30 min. Flies exposed to $\mathrm{ACV}$ $\mathrm{pH}=2(n=27)$ have 3.1-fold greater photoconversion compared to flies exposed to ACV $\mathrm{pH}=7(n=21)$. ****P $<0.0001$, Mann-Whitney $U$-test. 
low and in some low-activity circuits may be indistinguishable from background. Two improvements to CaMPARI have already been made to address these concerns: (1) an improved version of CaMPARI (CaMPARI2) that improves the brightness and contrast of red CaMPARI; and (2) an antibody that specifically detects the red CaMPARI chromophore, but not the green chromophore (Moeyaert et al., 2018). With these tools, CaMPARI freely diffuses through the cytoplasm and so subcellular information is lost. Recently the tool SynTagMa (Synaptic Tag for Mapping Activity) was created to label active synapses by fusing CaMPARI to synaptophysin or PSD95 and labeling presynaptic boutons and dendritic spines, respectively (Perez-Alvarez et al., 2019). This tool could be further adapted to more specifically label circuitry in adult Drosophila or any other species where transgenic methods allow the creation of viable transgenic animals. Additionally, pairing CaMPARI with other optogenetic tools will allow downstream tracing of active circuits. For example, using CsChrimson to activate a specific neuron while exposing a pan-neuronal expressed CaMPARI will allow an experimenter to trace any neurons excited by the neuron expressing CsChrimson.

Recently, another method for tracing neural activity in freely-moving flies was published by Grover et al. (2020) called Flyception2. This method makes use of a setup with a galvanometer mirror system that allows the fly to be simultaneously tracked and imaged with two separate cameras. This setup has the advantage of being able to directly link activity with behavior due to the two-camera setup but is a more complicated setup that may not be possible in all laboratories and still can only evaluate a single fly at once. Additionally, these flies require removal of the head cuticle that needs to be replaced with a window containing retroreflective beads before tracking and imaging is possible, while our method allows the head cuticle to remain intact. However, such a method could also work well with CaMPARI as it is set up for ratiometric quantification. While Flyception2 allows for behavior tracking while imaging neural activity, our method does not allow any behavioral tracking as it is presented here, though very open to modifications. Still, our method can detect broader changes in neural activity when the fly is presented with a stimulus and with the ability to evaluate these changes with higher resolution in later imaging.

Developing a method that allows whole-brain tracing of active circuitry in freely moving adult flies can allow CaMPARI to be

\section{REFERENCES}

Adhikari, P., Orozco, D., Randhawa, H., and Wolf, F. W. (2019). Mef2 induction of the immediate early gene $\mathrm{Hr} 38 / \mathrm{Nr} 4 \mathrm{a}$ is terminated by Sirt1 to promote ethanol tolerance. Genes Brain Behav. 18:e12486. doi: 10.1111/gbb.12486

Aharoni, D., and Hoogland, T. M. (2019). Circuit investigations with open-source miniaturized microscopes: past, present and future. Front. Cell. Neurosci. 13:141. doi: 10.3389/fncel.2019.00141

Ai, M., Min, S., Grosjean, Y., Leblanc, C., Bell, R., Benton, R., et al. (2010). Acid sensing by the Drosophila olfactory system. Nature 468, 691-695. doi: 10.1038/nature09537

Barnett, L. M., Hughes, T. E., and Drobizhev, M. (2017). Deciphering the molecular mechanism responsible for GCaMP6m's $\mathrm{Ca}^{2+}$-dependent change in fluorescence. PLoS One 12:e170934. doi: 10.1371/journal.pone.0170934 used in an unbiased and exploratory manner. It allows for the study of various behaviors from movement to sensory response to social interaction and beyond.

\section{DATA AVAILABILITY STATEMENT}

The datasets generated for this study are available on request to the corresponding author.

\section{AUTHOR CONTRIBUTIONS}

$\mathrm{KE}, \mathrm{MH}$, and GB designed the study. KE performed the experiments and analyzed the data. $\mathrm{KE}, \mathrm{MH}$, and $\mathrm{GB}$ wrote the article. All authors read and edited the manuscript.

\section{FUNDING}

This project was supported by grants from the Audrey and Theodore Geisel School of Medicine at Dartmouth, the National Institute of Health Pioneer grant 1DP1MH110234 (GB), the Defense Advanced Research Projects Agency grant HR001115-1-0002 (GB), and the National Institute of General Medical Sciences grant GM113132 (MH).

\section{ACKNOWLEDGMENTS}

We thank the Bloomington Drosophila Stock Center and Vienna Drosophila Resource Center for its stocks. We thank Vibhuti Rana for the careful reading of the manuscript and Balint Kacsoh and Madhu Sadanandappa for helpful comments as the project progressed. We would also like to thank Ann Lavanway and the Dartmouth College Department of Biological Sciences Imaging Facility for the use of the equipment and technical assistance as well as Zdenek Svindrych of the Molecular Interactions and Imaging Core for technical assistance.

\section{SUPPLEMENTARY MATERIAL}

The Supplementary Material for this article can be found online at: https:/www.frontiersin.org/articles/10.3389/fncir.2020.000 22/full\#supplementary-material.

Berridge, M. J., Lipp, P., and Bootman, M. D. (2000). The versatility and universality of calcium signalling. Nat. Rev. Mol. Cell Biol. 1, 11-21. doi: $10.1038 / 35036035$

Bohra, A. A., Kallman, B. R., Reichert, H., and VijayRaghavan, K. (2018). Identification of a single pair of interneurons for bitter taste processing in the Drosophila brain. Curr. Biol. 28, 847.e3-858.e3. doi: 10.1016/j.cub.2018. 01.084

Chen, T.-W., Wardill, T. J., Sun, Y., Pulver, S. R., Renninger, S. L., Baohan, A., et al. (2013). Ultrasensitive fluorescent proteins for imaging neuronal activity. Nature 499, 295-300. doi: 10.1038/nature12354

Cole, A. J., Saffen, D. W., Baraban, J. M., and Worley, P. F. (1989). Rapid increase of an immediate early gene messenger RNA in hippocampal neurons by synaptic NMDA receptor activation. Nature 340, 474-476. doi: 10.1038/340 $474 \mathrm{a} 0$ 
de Groot, A., van den Boom, B. J., van Genderen, R. M., Coppens, J., van Veldhuijzen, J., Bos, J., et al. (2020). NINscope, a versatile miniscope for multiregion circuit investigations. eLife 9:e49987. doi: 10.7554/eLife.49987

Fields, R. D., Eshete, F., Stevens, B., and Itoh, K. (1997). Action potentialdependent regulation of gene expression: temporal specificity in $\mathrm{Ca}^{2+}$, cAMP-responsive element binding proteins and mitogen-activated protein kinase signaling. J. Neurosci. 17, 7252-7566. doi: 10.1523/jneurosci.17-1907252.1997

Fosque, B. F., Sun, Y., Dana, H., Yang, C.-T., Ohyama, T., Tadross, M. R., et al. (2015). Labeling of active neural circuits in vivo with designed calcium integrators. Science 347, 755-760. doi: 10.1126/science.1260922

Fujita, N., Nagata, Y., Nishiuchi, T., Sato, M., Iwami, M., and Kiya, T. (2013). Visualization of neural activity in insect brains using a conserved immediate early gene, Hr38. Curr. Biol. 23, 2063-2070. doi: 10.1016/j.cub.2013.08.051

Grover, D., Katsuki, T., Li, J., Dawkins, T. J., and Greenspan, R. J. (2020). Imaging brain activity during complex social behaviors in Drosophila with Flyception2. Nat. Commun. 11:623. doi: 10.1038/s41467-020-14487-7

Guzowski, J. F., McNaughton, B. L., Barnes, C. A., and Worley, P. F. (1999). Environment-specific expression of the immediate-early gene Arc in hippocampal neuronal ensembles. Nat. Neurosci. 2, 1120-1124. doi: $10.1038 / 16046$

Jia, H., Rochefort, N. L., Chen, X., and Konnerth, A. (2011). in vivo two-photon imaging of sensory-evoked dendritic calcium signals in cortical neurons. Nat. Protoc. 6, 28-35. doi: 10.1038/nprot.2010.169

Link, W., Konietzko, U., Kauselmann, G., Krug, M., Schwanke, B., Frey, U., et al. (1995). Somatodendritic expression of an immediate early gene is regulated by synaptic activity. Proc. Natl. Acad. Sci. U S A 92, 5734-5738. doi: 10.1073/pnas. 92.12.5734

Looger, L. L., and Griesbeck, O. (2012). Genetically encoded neural activity indicators. Curr. Opin. Neurobiol. 22, 18-23. doi: 10.1016/j.conb.2011.10.024

Manjila, S. B., Kuruvilla, M., Ferveur, J.-F., Sane, S. P., and Hasan, G. (2019). Extended flight bouts require disinhibition from GABAergic mushroom body neurons. Curr. Biol. 29, 283.e5-293.e5. doi: 10.1016/j.cub.2018.11.070

Moeyaert, B., Holt, G., Madangopal, R., Perez-Alvarez, A., Fearey, B. C., Trojanowski, N. F., et al. (2018). Improved methods for marking active neuron populations. Nat. Commun. 9:4440. doi: 10.1038/s41467-018-06935-2

Palmer, A. E., and Tsien, R. Y. (2006). Measuring calcium signaling using genetically targetable fluorescent indicators. Nat. Protoc. 1, 1057-1065. doi: $10.1038 /$ nprot.2006.172

Paredes, R. M., Etzler, J. C., Watts, L. T., Zheng, W., and Lechleiter, J. D. (2008). Chemical calcium indicators. Methods 46, 143-151. doi: 10.1016/j.ymeth.2008. 09.025

Patel, A. A., and Cox, D. N. (2017). Behavioral and functional assays for investigating mechanisms of noxious cold detection and multimodal sensory processing in Drosophila Larvae. Bio. Protoc. 7:e2388. doi: 10.21769/ BioProtoc. 2388
Perez-Alvarez, A., Fearey, B. C., O’Toole, R. J., Yang, W., Arganda-Carreras, I., Lamothe-Molina, P. J., et al. (2019). Freeze-frame imaging of synaptic activity using SynTagMA. bioRxiv [Preprint]. doi: 10.1101/538041

Ramdya, P., Schneider, J., and Levine, J. D. (2017). The neurogenetics of group behavior in Drosophila melanogaster. J. Exp. Biol. 220, 35-41. doi: 10.1242/jeb. 141457

Riemensperger, T., Pech, U., Dipt, S., and Fiala, A. (2012). Optical calcium imaging in the nervous system of Drosophila melanogaster. Biochim. Biophys. Acta 1820, 1169-1178. doi: 10.1016/j.bbagen.2012.02.013

Rodrigues, V., and Buchner, E. (1984). $\left[{ }^{3} \mathrm{H}\right] 2$-Deoxyglucose mapping of odor-induced neuronal activity in the antennal lobes of Drosophila melanogaster. Brain Res. 324, 374-378. doi: 10.1016/0006-8993(84) 90053-2

Sanders, J. I., and Kepecs, A. (2014). A low-cost programmable pulse generator for physiology and behavior. Front. Neuroeng. 7:43. doi: 10.3389/fneng.2014.00043

Schindelin, J., Arganda-Carreras, I., Frise, E., Kaynig, V., Longair, M., Pietzsch, T., et al. (2012). Fiji: an open-source platform for biological-image analysis. Nat. Methods 9, 676-682. doi: 10.1038/nmeth.2019

Sha, F., Abdelfattah, A. S., Patel, R., and Schreiter, E. R. (2019). rsCaMPARI: an erasable marker of neuronal activity. bioRxiv [Preprint]. doi: 10.1101/798298

Suh, G. S. B., Wong, A. M., Hergarden, A. C., Wang, J. W., Simon, A. F., Benzer, S., et al. (2004). A single population of olfactory sensory neurons mediates an innate avoidance behaviour in Drosophila. Nature 431, 854-859. doi: 10.1038/nature02980

Sun, X. R., Badura, A., Pacheco, D. A., Lynch, L. A., Schneider, E. R., Taylor, M. P., et al. (2013). Fast GCaMPs for improved tracking of neuronal activity. Nat. Commun. 4:2170. doi: 10.1038/ncomms3170

Tian, L., Hires, S. A., Mao, T., Huber, D., Chiappe, M. E., Chalasani, S. H., et al. (2009). Imaging neural activity in worms, flies and mice with improved GCaMP calcium indicators. Nat. Methods 6, 875-881. doi: 10.1038/ nmeth.1398

Wu, J. S., and Luo, L. (2006). A protocol for dissecting Drosophila melanogaster brains for live imaging or immunostaining. Nat. Protoc. 1, 2110-2115. doi: $10.1038 /$ nprot.2006.336

Conflict of Interest: The authors declare that the research was conducted in the absence of any commercial or financial relationships that could be construed as a potential conflict of interest.

Copyright (c) 2020 Edwards, Hoppa and Bosco. This is an open-access article distributed under the terms of the Creative Commons Attribution License (CC BY). The use, distribution or reproduction in other forums is permitted, provided the original author(s) and the copyright owner(s) are credited and that the original publication in this journal is cited, in accordance with accepted academic practice. No use, distribution or reproduction is permitted which does not comply with these terms. 